\title{
Different types of glomerulopathic light chains interact with mesangial cells using a common receptor but exhibit different intracellular trafficking patterns
}

\author{
Jiamin Teng ${ }^{1}$, William J. Russell ${ }^{1}$, Xin $\mathrm{Gu}^{1}$, James Cardelli², M Lamar Jones ${ }^{1}$ \\ and Guillermo A. Herrera ${ }^{1,3,4}$ \\ ${ }^{1}$ Department of Pathology; ${ }^{2}$ Department of Microbiology; ${ }^{3}$ Department of Cell Biology and Anatomy and \\ ${ }^{4}$ Department of Medicine, Louisiana State University Health Sciences Center, Shreveport, LA, USA
}

\begin{abstract}
Patients with plasma cell dyscrasias may have circulating light chains (LCs), some of which are nephrotoxic. Nephrotoxic LCs can affect the various renal compartments. Some of these LCs may produce predominantly proximal tubular damage, while others are associated with distal nephron obstruction (the so-called 'myeloma kidney'). Both these are considered tubulopathic (T) LCs. A receptor has been found in proximal tubular cells (cubilin/megalin complex), which mediates the absorption of LCs and is involved in the pathogenesis of tubulopathies that occurs in these patients. Another group of nephrotoxic LCs is associated with glomerular damage and these are considered as glomerulopathic (G). These patients with G-LCs may develop ALamyloidosis (AL-Am) or LC deposition disease (LCDD). Recent evidence indicates that the physicochemical characteristics (amino-acid composition and conformation of the variable region) of a given nephrotoxic LC may be the most significant factor in determining the type and location of renal damage within the nephron. Other factors may also be involved, including yet uncharacterized host factors that may include genetic polymorphism, among others. Interestingly, the amount of LC production by the clone of plasma cells does not correlate directly with the severity of the renal alterations. Understanding the nature of the interactions between G-LCs and mesangial cells (MCs) is crucial to define key steps that may be targeted for therapeutic purposes. Experimental studies have delineated important aspects pertaining to interactions between G-LCs and MCs, indicating that these interactions are receptor mediated. The data presented in the current study support a single receptor present on MCs for both LCDD and AL-LCs, as clearly demonstrated with competition and colocalization immunofluorescence (IF) studies. This receptor resides in caveolae present on the plasma membrane of HMCs and is overexpressed when HMCs are incubated with G-LCs but not TLCs. Caveolae play a fundamental role in receptor-mediated endocytosis, a crucial process in the internalization of AL-LCs and amyloidogenesis. LC internalization is clathrin mediated. The data also indicate that intracellular trafficking in MCs is different for AL-LCs and LCDD-LCs. AL-LCs are delivered to the mature lysosomal compartment where amyloid formation occurs. LCDD-LCs alter mesangial function and phenotype by interacting with the MC surface membranes through similar receptors as the AL-LCs. The data also demonstrated that cubilin and megalin were absent on MCs, so the receptor involved is different from the one already characterized in the proximal tubules.
\end{abstract}

Laboratory Investigation (2004) 84, 440-451, advance online publication, 1 March 2004; doi:10.1038/labinvest.3700069

Keywords: myeloma; light chains; plasma cell dyscrasia; mesangial cells; light-chain deposition disease; AL-amyloidosis; receptor

While the majority of the nephrotoxic light chains (LCs) produce tubular damage, approximately

Correspondence: GA Herrera, Department of Pathology, Louisiana State University Health Sciences Center, 1501 Kings Highway, Shreveport, LA, 71130, USA.

E-mail: gherre@lsuhsc.edu

Received 17 June 2003; accepted 05 November 2003; published online 1 March 2004
$30 \%$ are primarily associated with glomerular pathology. Binding data relating to the tubular receptor for LCs have suggested that cubilin first binds to megalin on cell membranes, where the cubilin-megalin complex is internalized simultaneously for endosomal trafficking in the proximal tubules. ${ }^{1}$ The pathogenic effects of glomerulopathic (G)-LCs are centered in the mesangium. 
G-LCs alter mesangial homeostasis. Their effects on mesangial cells (MCs) are variable depending on the biochemical composition of the variable region of the LCs resulting in two well-characterized disease processes: Al-amyloidosis (AL-Am) and LC depostion disease (LCDD). ${ }^{2-4}$ The LCs obtained from the urine of patients with these disorders are referred as AL and LCDD-LCs, respectively. Nephrotoxic LCs may either affect both the tubular interstitial and glomerular compartments in a given case or may restrict their damage to one compartment, or the two glomerulopathies may coexist. As such, some LCs may elicit their pathologic effects producing a combination of lesions (ie proximal tubular damage and distal nephron obstruction in the tubular interstitial compartment or LCDD and ALAm in the glomerular compartment). The majority of the nephrotoxic LCs, however, produce a specific pattern of renal damage confined to one renal compartment and restricted to a specific lesion. Vascular alterations also occur in a significant number of cases associated with AL-Am and in some cases with LCDD.

The present paper focuses on the interactions between G-LCs and MCs and how these produce pathologic alterations. The study aimed at elucidating whether a specific receptor was shared by all G-LCs or more than one receptor was involved, leading to AL-Am and LCDD. Immunogold labeling studies at the ultrastructural level, coimmunolocalization using IF, binding competition and crosslinking studies demonstrated the presence of a single receptor for both types of G-LCs. This receptor must act differently when involved in LC-MC interactions associated with AL-Am and LCDD, as the final result of the pathogenetic effects is entirely different. In the case of AL-LCs, endocytosis is a crucial step in the formation of amyloid and resultant pathologic changes leading to mesangial matrix degradation and replacement of normal matrix by amyloid fibrils. In contrast, in LCDD-LC interactions with MCs, internalization is not an important initial process and the alterations in mesangial homeostasis dictated by the G-LCs appear to occur primarily at the MC surface. ${ }^{5}$ The receptor modulating and regulating LC-MC interactions is different from the receptor identified for LCs in the proximal tubular cells. The main objectives of the work reported in this paper are to confirm the existence of such receptor-mediating G-LC-MC pathologic events and to define whether AL and LCDD-LCs exhibit similar or different intracellular trafficking pathways in MCs. Further studies to characterize this receptor fully are currently being conducted.

\section{Materials and methods}

\section{Protein purification}

G-LCs were purified from the urine of patients who had AL-Am, LCDD and myeloma cast nephropathy proven by renal biopsy. The LCs were obtained and purified from the urine of these patients using ammonium sulfate precipitation and ion exchange chromatography, as described previously. ${ }^{6}$

Briefly, affinity chromatography consisted of passing urine over sepharose CL-4B columns to which either goat anti-human $\lambda$ or $\kappa$ antibodies have been conjugated. Free plus bound $\lambda$ or $\kappa$ LCs were eluted from the columns with 0.1 glycine, $\mathrm{pH} 2.2$. The purity of the LC was checked on immunodiffusion plates and anti-IgG and anti-IgA affinity columns were used to remove contaminating IgG and IgA. When purified, the LC migrated as a single band when resolved by zone electrophoresis on agarose gels using a $50 \mathrm{mM}$ barbital buffer, $\mathrm{pH}$ 8.6, and consisted entirely of either free $\lambda$ or $\kappa$ LC as determined by immunofixation. Purified LCs were concentrated, dialyzed against $0.9 \mathrm{NaCl}$, filtered through a $0.2-\mathrm{m}$ membrane and stored aseptically at $1 \mathrm{mg} / \mathrm{ml}$ at $4^{\circ} \mathrm{C}$. The extracted LCs had the same composition as those circulating in the serum of the corresponding patients.

\section{Human Mesangial Cell (HMC) Culture}

HMCs were cultured following the standard method described by Harper et al. ${ }^{7}$ Briefly, the cortex of surgically removed human kidneys was separated and glomerular MCs were isolated by sieving the cortex through stainless-steel screens and a series of different pore size nylon sieves. The cells were plated for culture in RPMI 1640 containing 20\% fetal calf serum and penicillin, streptomycin and fungizone. For some experiments, the HMCs were grown on coverslips placed on $20 \mathrm{~mm}$ culture plates. Ultrastructural examination and immunocytochemistry staining with immunoreactivity for musclespecific antigen (HHF-35 antigen) and vimentin and lack of staining for keratin and factor VIII were used to determine that these cells were a homogeneous population of HMCs. Passage 5-6 HMCs, but not older, were grown on RPMI 1640 medium containing $15 \%$ fetal bovine serum (FBS) until confluent. Once confluent, HMCs were cultured in low serum (0.5\% FBS) RPMI 1640 medium for $48 \mathrm{~h}$ to render the cells quiescent prior to the experiments.

\section{Immunofluorescence (IF)}

HMCs grown on coverslips incubated with one or two different G-LCs at the same time (LCDD and ALLCs) and T-LCs were fluorescence tagged for $\kappa$ and or $\lambda$ LCs in order to label the various LCs and caveolin-1 to determine whether the G-LCs colocalized to the same surface MC areas and whether there was any attraction for the TLC to the MCs membranes or competition with the G-LCs. A direct immunofluorescence stain procedure was performed. ${ }^{8}$ A polyclonal antibody to caveolin-1 (Santa Cruz Biotechnology, Santa Cruz, CA, USA) was used 
at a dilution of $1 / 50$ and those to $\kappa$ and $\lambda$ at a dilution of $1 / 200$. LCs were labeled with either fluorescein, rhodamine or Texas red. Areas of colocalization were detected by a yellow signal indicating merging of green and red.

In other studies, fluorescent-labeled polyclonal antibodies to Rabs 3, 5, 7 and 9 (Santa Cruz Biotechnology) each at $1 / 50$ to $1 / 75$ dilutions were used to study trafficking of LCs in MCs. Also, fluorescent-labeled monoclonal antibodies to cathepsin D and lysosomal-associated membrane protein (LAMP) (Santa Cruz Biotechnology) at a 1/50 dilution were used to detect whether colocalization with the G-LCs occurred, and to identify possible differences between AL and LCDD-LCs trafficking patterns. Finally, a fluorescent-labeled monoclonal antibody to clathrin (dilution 1/25) was employed to evaluate morphologically the role of clathrin in LC endocytosis by MCs. All these antibodies were used to label HMCs growing on coverslips incubated with AL, LCDD and T-LCs, as well as MCs alone (not incubated with LCs) to evaluate differences in LCMC interactions, LC internalization and LC trafficking pathways in MCs. All fluorescent-labeled sections were examined using a BX60 fluorescence Olympus microscope (C Squared Co., Tamarac, FL, USA).

\section{Immunohistochemistry}

HMCs grown on coverslips were grown unexposed to LCs or incubated with LCDD and AL-LCs for $1 \mathrm{~h}$ and then immunostained for cubilin and megalin in order to determine whether MCs expressed these two receptors. Immunoperoxidase staining was performed using the Universal streptavidin-biotinlabeled horseradish peroxidase (HRP) system (Dako Corporation, Carpinteria, CA, USA) for 20 min using a steamer and following a well-tested procedure. ${ }^{9}$ The specimens were placed in $3 \%$ hydrogen peroxide (Vector Laboratories, Burlingame, CA, USA) for $5 \mathrm{~min}$ to block endogenous peroxidase and rinsed in three changes of Dako automation buffer for 2 min each. The goat antibodies to cubilin and megalin (Santa Cruz Biotechnology) at a dilution of 1/500 were applied to the HMCs growing on the cover-slips for $30 \mathrm{~min}$ at room temperature. The coverslips were then rinsed in Dako automation buffer for four changes, $2 \mathrm{~min}$ each. The negative controls were generated by replacing the primary antibody with nonspecific rabbit or mouse IgG. The secondary biotinylated bovine anti-goat IgG-HRP (Santa Cruz) antibody was applied to the coverslips for $15 \mathrm{~min}$ at room temperature and rinsed with Dako automation fluid, four changes for $2 \mathrm{~min}$ each. The diaminobenzidine chromogen (Dako Co.) was applied to the tissue sections for 5-10 min at room temperature and rinsed with Dako automation fluid, three changes for 2 min each. Hematoxylin (Biogenex Laboratories, San Ramon, CA, USA) was applied to the slides for $1 \mathrm{~min}$, rinsed with distilled water, two changes and placed in Dako automation buffer for $5 \mathrm{~min}$. The coverslips were then rinsed with distilled water, dehydrated with two changes of $95 \%$ alcohol, $100 \%$ alcohol, xylene and sealed with a permanent mounting media.

\section{Ultrastructural Labeling Studies}

HMCs incubated with G and T-LCs for 1, 3, 6 and $12 \mathrm{~h}$ were processed for routine ultrastructural evaluation and immunogold labeling. The specimens were fixed in Carson-Millonig solution (Polysciences Inc., Warrington, PA, USA), embedded in epoxy or LR White resins (London Resin, Surrey, UK) and thick sectioned for light microscopic survey. Toluidine blue-stained thick sections were used to select areas for the preparation of thin sections. Once appropriate areas were identified, thin sections were placed on uncoated copper grips, stained with uranyl acetate and lead citrate and examined ultrastructurally. For immunoelectron microscopy, thin sections were placed on uncoated nickel grids. The labeling procedure has been described previously. ${ }^{10}$ Briefly, grids were incubated in 3\% normal goat serum (Vector Laboratories) and in polyclonal antibodies to $\kappa$ and $\lambda$ LCs (Dako CO) at $4^{\circ} \mathrm{C}$ for $16 \mathrm{~h}$. Both antibodies were used at 1/200 and $1 / 400$ dilutions. After washing, the grids were incubated with $20 \mathrm{~nm}$ gold-labeled antiserum to rabbit IgG (Janssen Pharmaceutical, Beerse, Belgium) diluted at $1 / 20$ for $1 \mathrm{~h}$. The grids were finally poststained with uranyl acetate and lead citrate. The labeled grids were examined under a Philips CM12 transmission electron microscope (NV Philips). Positive controls consisted of renal biopsies from patients with $\kappa$-LCDD and $\lambda$-AL-amyloidosis. Negative controls were represented by two renal biopsies with minimal change glomerulopathy.

\section{Binding Studies}

Using Iodo-Gen-coated tubes (Pierce Biotechnology, Rockford, IL, USA), $500 \mu \mathrm{g}$ of either LCDD, AL or T-LC were reacted with $0.5 \mathrm{mCi} .{ }^{125} \mathrm{I}$ in PBS for $5 \mathrm{~min}$ at $4{ }^{\circ} \mathrm{C} .{ }^{125} \mathrm{I}$ (Amersham, Arlington Heights, IL, USA) labeled LC was separated from unincorporated free ${ }^{125}$ I by passage over a Sephadex G-25 column (PD-10 column form Pharmacia, Pistacaway, NJ, USA). HMCs in 24-well plates grown to confluence in RPMI 1640 with 15\% FBS were made quiescent by culture for an additional $24 \mathrm{~h}$ in low serum $(0.5 \%$ FBS) RPMI. These quiescent HMCs were then rinsed twice with $\mathrm{pH} 5.0$ HBSS containing $10 \mathrm{mM}$ acetic acid prior to $1 \mu \mathrm{g}$ of ${ }^{125} \mathrm{I}$-LC being added to $1 \mathrm{ml}$ of the same pH 5.0 HBSS buffer with $0.1 \%$ BSA and $0.05 \%$ $\mathrm{NaN}_{3}$ per well of HMCs at $4{ }^{\circ} \mathrm{C}$ or room temperature $\left(22^{\circ} \mathrm{C}\right)$. At $0,15,30,45$ and $60 \mathrm{~min}$, the ${ }^{125} \mathrm{I}-\mathrm{LC}$ containing HBSS was removed and the HMCs were rinsed three times with $\mathrm{pH}$ 5.0 HBSS at the same 
temperature. A measure of $500 \mu \mathrm{l}$ of $\mathrm{N} \mathrm{NaOH}$ was added to lyse the cells and radioactivity determined. Results were represented using bar graphs and Scatchart plots to illustrate the findings clearly.

\section{Competition Studies}

Binding competition studies using two LCs at the same time (one radiolabeled with ${ }^{125} \mathrm{I}$ and the other one not radiolabeled) were also performed. The two LCs were simultaneously placed on the quiescent MCs as described above. G (LCDD and AL) and T-LCs were used for the competition studies. The concentration of the hot (radiolabeled) LC was maintained the same, while the concentration of the cold LC was increased to test whether competition for the same receptor and less binding of the 'hot' LC to MCs (indicating competition) occurred. Both $\mathrm{G}$ and T-LCs were used to determine whether differences in binding and competition would occur.

\section{Colocalization Studies}

IF stains for caveolin-1 and the various G and T-LCs were performed on HMCs grown on coverslips separately and simultaneously to detect whether colocalization occurred. Also, immunofluorescence staining for caveolin-1 was performed in MCs growing on coverslips incubated with fluorescence-tagged $\mathrm{G}$ and T-LCs. The polyclonal antibodies employed against $\kappa$ and $\lambda$ were diluted to $1 / 200$ and the polyclonal antibody to caveolin-1 was employed at a 1/50 dilution. MCs not incubated with LCs were used as controls. IF staining was also used to colocalize LCs and clathrin as well as LCs and LAMP and cathepsin D, using fluorescein and Texas red as markers.

\section{Dynamic Studies in pH/temperature-Regulated System}

A chamber, temperature and $\mathrm{pH}$ controlled system to maintain the MCs viable was employed to study in a dynamic real-time manner the course of the LCMC interactions. A 24-well plate was modified to keep water flowing between the wells at a constant temperature $\left(37^{\circ} \mathrm{C}\right)$. A $5 \% \quad \mathrm{CO}_{2}$ air flow at 0.1 to $0.2 \mathrm{~cm}^{3} / \mathrm{s}$ was used to keep a constant $\mathrm{pH}$ in the chamber. The chamber maintained the HMCs in incubator-like conditions on a microscope platform to visualize microscopically the G-LCs from initial binding to MCs to final intracellular location in order to determine intracellular trafficking pathways. LysoTracker probes (Molecular Probes Inc., Eugene, OR, USA) consisting of weakly basic amines selectively accumulating in cellular compartments with low internal $\mathrm{pH}$ were used to delineate the mature lysosomal compartment. The LCs of interest were labeled using direct immunofluorescence and placed in the medium. The labeled G-LCs were followed as they migrated to bind to the MCs, became internalized and were delivered to the mature lysosomal compartment (AL-LCs). The trafficking routes of various LCs were then monitored in a real-time manner using a BX60 fluorescence Olympus microscope (C Squared Co., Tamarac, FL, USA).

\section{G-LC-MC Membrane Crosslinking Studies}

Using 3-3'-dithiobis (sulfosuccinimidyl propionateDTSSP) (Pierce Biotechnology), a water-soluble membrane-impermeable thio cleavable cross-linker, it was possible to crosslink the different types of $\mathrm{G}$-LCs to the surface of quiescent HMCs. Confluent monolayers of quiescent HMCs in $100 \mathrm{~mm}$ dishes were incubated for $30 \mathrm{~min}$ at room temperature with $10 \mu \mathrm{g} / \mathrm{ml}$ medium G-LCs added to RPMI 1640 medium, pH 5.0 containing $0.5 \%$ FBS and $10 \mathrm{mM}$ acetic acid. The medium was acidified to inhibit clathrin mediated internalization of the LCs (primarily the AL-LC). Afterwards, the cells were quickly rinsed twice with borate-buffered saline (10 mM Na $\mathrm{Na}_{2} \mathrm{~B}_{4}, 150 \mathrm{mM} \mathrm{NaCl}, \mathrm{pH}$ 8.0) and incubated at RT with $1 \mathrm{mM}$ DTSSP in the same buffer. After $30 \mathrm{~min}$, the crosslinking reaction was stopped by rinsing the cells four times with $20 \mathrm{mM}$ Trisbuffered saline (TBS). The cells were then scraped from the dishes, pelleted by centrifugation at $1000 \mathrm{rpm}(210 \times g)$ for $5 \mathrm{~min}$ and lysed in $500 \mu \mathrm{l}$ of a modified Laemmli buffer, $1 \times$ NuPAGE sample buffer (Invitrogen, Carlsbad, CA, USA) The lysate was cleared by centrifugation at $10000 \mathrm{~g}$ for $10 \mathrm{~min}$ at $4{ }^{\circ} \mathrm{C}$. Proteins contained within the lysate were resolved by SDS-PAGE under nonreducing conditions on NuPAGE 4-12\% Bis-Tris polyacrylamide gels using NuPAGE MOPS running buffer. The resolved bands were electrophoretically transferred onto a PVDF membrane. The membrane was blocked with $5 \%$ mild protein in TBS and proteins to which the LC was crosslinked were identified by using rabbit anti-human $\kappa$ or $\lambda$ at a dilution or 1:2000 (Dako Co.) as primary antibody, donkey anti-rabbit HRP conjugate at a 1:1500 dilution as secondary antibody and ECL (Pharmacia) as chemiluminescent substrate. A Coomassie blue stain was then performed to better define the shifted bands observed.

\section{Statistical Analysis}

Results are reported as mean \pm s.e.m. Statistical significance was determined by one-way ANOVA followed by post hoc comparisons performed with a Bonferroni/Dunn test using the SPSS statistical analysis package. A $p$-value of $<0.05$ was considered statistically significant. Such statistical significance is signified in the appropriate grafts with an asterisk (*). 


\section{Results}

\section{Cubilin and Megalin Are Not Present on HMCs}

Immunohistochemical studies performed on HMCs failed to reveal expression of cubilin or megalin. Even when HMCs were incubated with G-LCs (LCDD and AL-LCs), cubilin and megalin expression did not occur.

G-LCs Interact with Small Invaginations of the Plasma Membrane of HMCs With Ultrastructural Features of Caveolae and Expressing Caveolin-1 as Detected by Immunohistochemistry

Ultrastructural evaluation revealed small invaginations on the cell membrane of HMCs with the appearance of caveolae (Figure 1a, arrows) immunoreactive for caveollin-1 that colocalized with AL and LCDD-LCs (Figure 2, top). The number of these structures increased significantly when the HMCs were incubated with G but not T-LCs (Figure 1a, arrows). The number of caveolae was approximately seven to 10 times more when MCs were incubated with G-LCs than in MCs unexposed to G-LCs. As expected, caveolin-1 immunoreactivity increased dramatically when HMCs were incubated with GLCs when compared with HMCs not incubated with LCs or treated with T-LCs (Figure 2, bottom). Using ultrastructural labeling, G-LCs were noted to interact with the surface of HMCs where caveolae were identified (Figure 1b).

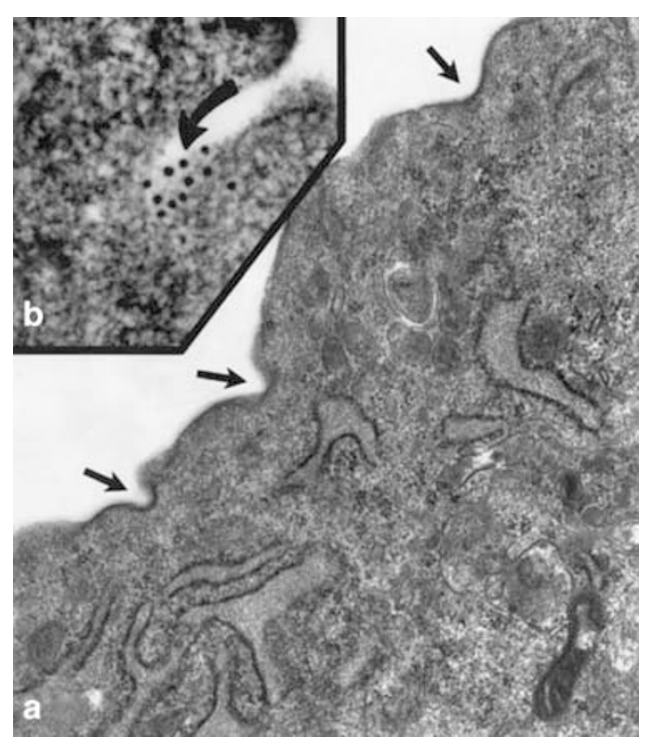

Figure 1 (a) HMCs incubated with G-LCDD-LCs for $24 \mathrm{~h}$. Transmission electron microscopy. Uranyl acetate and lead citrate stain A $\times 22500$. B $\times 35000$-Immunogold labeling for $\kappa \mathrm{LC}, 20 \mathrm{~nm}$ gold particles. Caveolae in various stages of development (arrows) increased in numbers significantly $(7-10 \times)$ when HMCs were incubated with G-LCs. (b) demonstrates $\kappa$ LCs intimately interacting with caveolae (cup-shaped surface indentation) at the HMC membrane.
Co-localization of G-LCs and caveolin-1 on HMCs
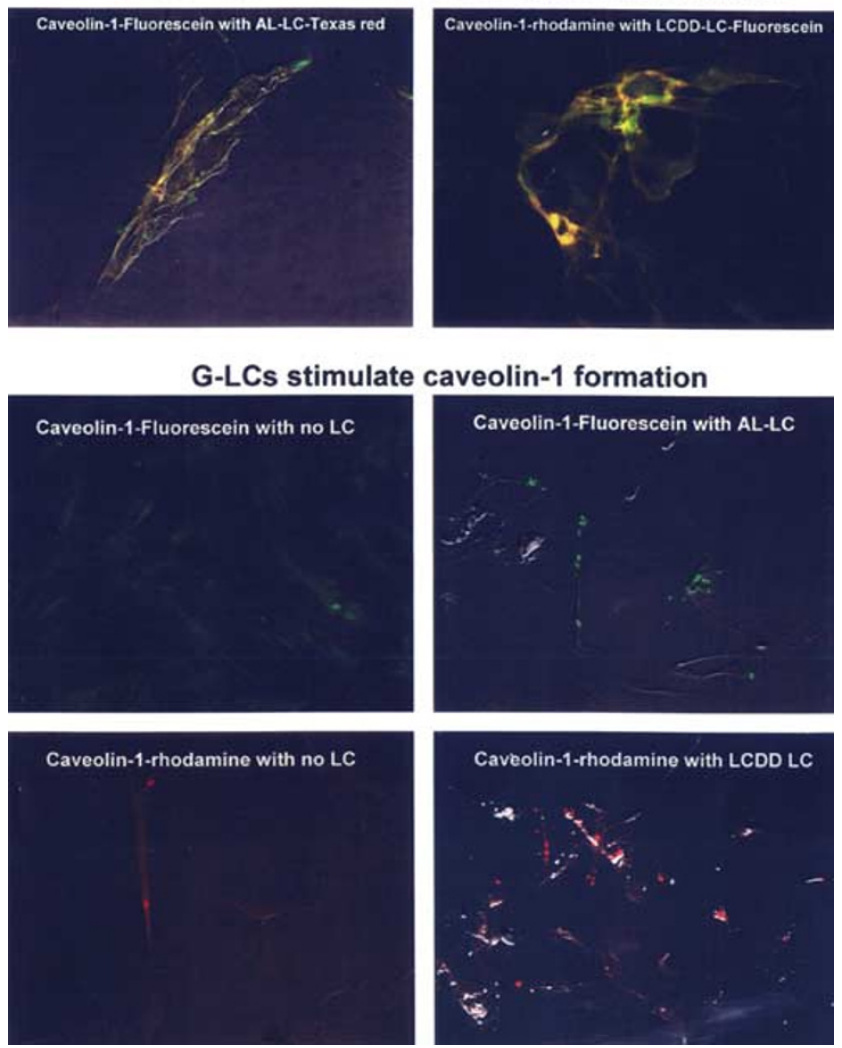

Figure 2 X350 HMCs incubated with LCDD and AL-LCs for 30 min. IF staining for caveolin-1. Fluorescent microscopy. (Top panel) AL and bottom panel LCDD-LC. (Bottom panel) Comparison of caveolin-1 staining in HMCs with no LC (left) and incubated with AL and LCDD-LCs (right, top and bottom, respectively). In right panels, top and bottom, note marked increased in caveolin-1 staining when HMCs are incubated with either AL or LCDD-LCs, as compared with left panels.

\section{G-LCs but Not T-LCs Bind to HMCs}

Both LCDD and AL-LCs showed significant binding to HMCs, while T-LCs did not when statistically compared with control (Figure 3). Maximum binding occurred at $30 \mathrm{~min}$ postincubation with the G-LCs (Figure 4, left). The binding of both types of G-LCs was also dose dependent to a point where saturation was achieved (Figure 4, right).

\section{LCDD and AL-LCs Competed for the Same Receptor on HMCs}

G-LCs (LCDD and AL-LCs) competed for binding to HMCs (Figure 5, upper and lower panels, left side), while T-LCs did not compete (Figure 5, upper and lower panels, right side). When two G-LCs were tested (one radiolabeled), competition was clearly shown with a statistically significant decrease in binding as the concentration of the nonradiolabeled LCs was increased. Scatchard plots were prepared to illustrate the findings (Figure 6). 


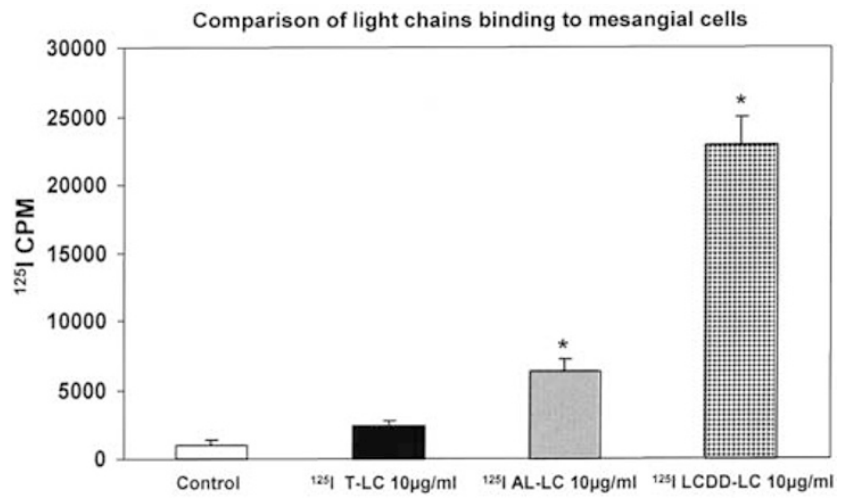

Figure 3 HMCs incubated with ${ }^{125}$ I radiolabeled T, AL and LCDDLCs, as well as control cells (not incubated with LC) for $30 \mathrm{~min}$. $\mathrm{AL}$ and LCDD-LCs binding to MCs is statistically significant compared to those of control cells or T-LCs. Binding of LCDD-LC to MCs is approximately $4-5 \times$ that of AL-LCs.

\section{G-LCs Colocalized at the Surface of HMCs}

Both G-LCs colocalized in specific areas on the surface of HMCs (Figure 7). Incubation with T-LCs failed to reveal colocalization with the G-LCs. In fact, no specific binding of T-LCs to HMCs could be demonstrated.

\section{LC Endocytosis and Trafficking in MCs}

Colocalization of G-LC with clathrin was clearly seen in morphologic studies (Figure 8), emphasizing the key role that clathrin plays in interactions of G-LCs and HMCs and in endocytosis of AL-LCs. Clathrin and LCDD-LCs virtually only colocalized at the surface and periphery of MCs very close to the cell membranes, since these LCs do not traffic to the mature endosomal system. In contrast, there was no
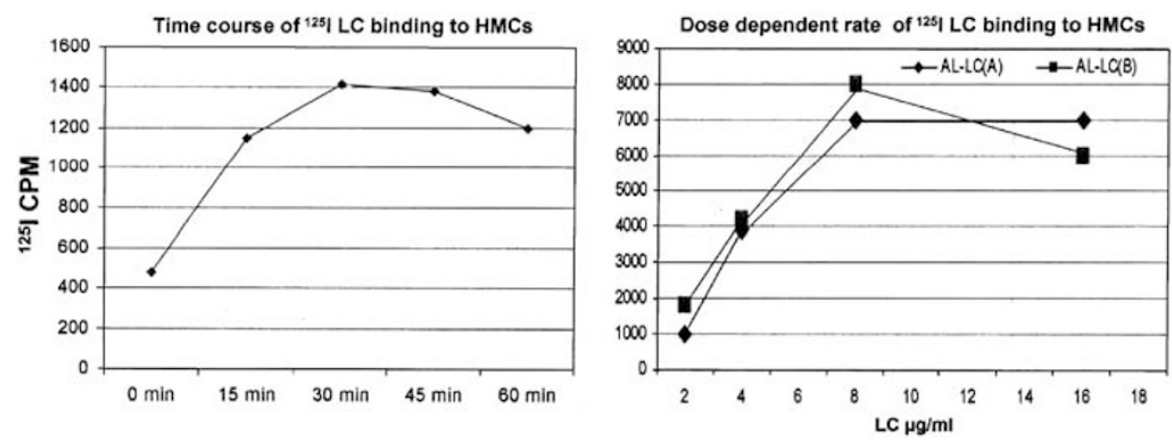

Figure 4 HMCs incubated with ${ }^{125}$ I radiolabeled AL and LCDD-LCs for varying amounts of time and varying concentrations. Time- and dose-dependent saturation is shown. Both AL and LCDD-LCs revealed maximum binding at 30 min postincubation with MCs.

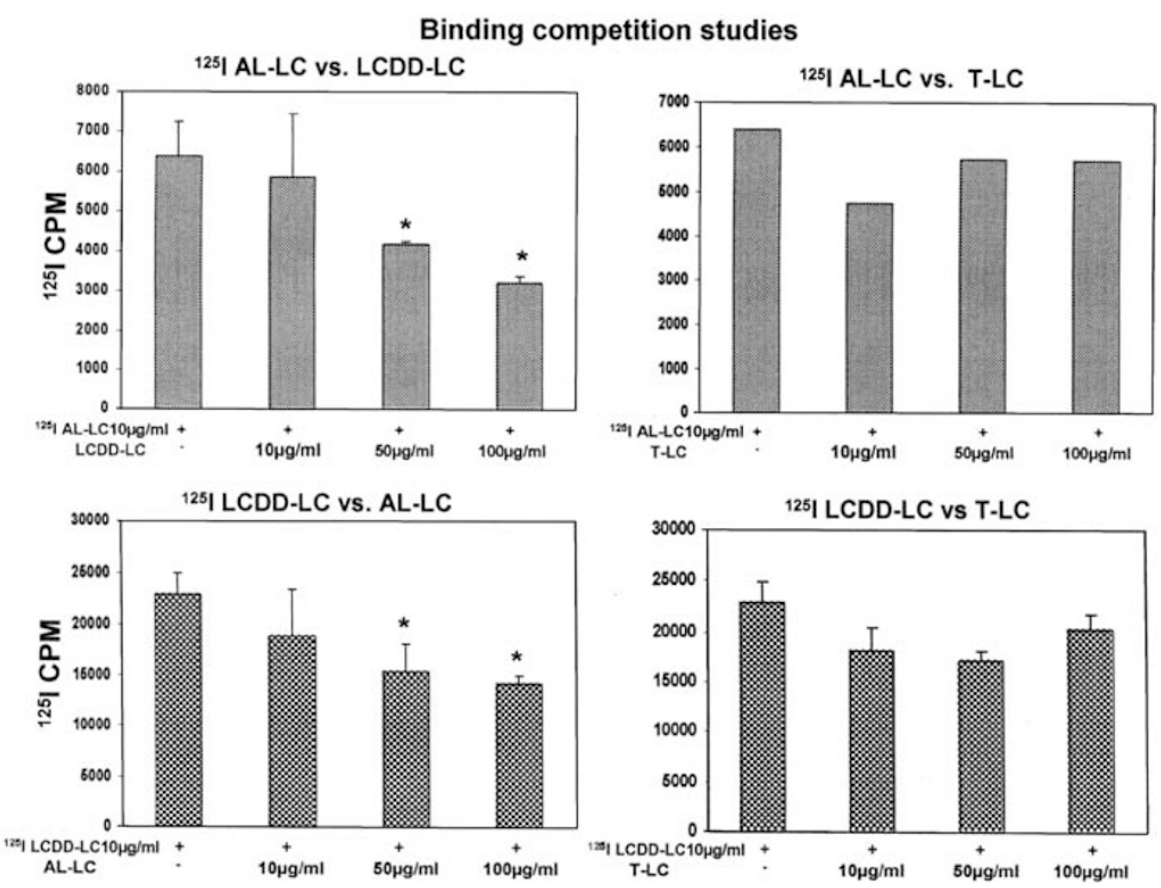

Figure 5 Scatchard plots illustrating the relationship between LCs bound to MCs and free LCs. 

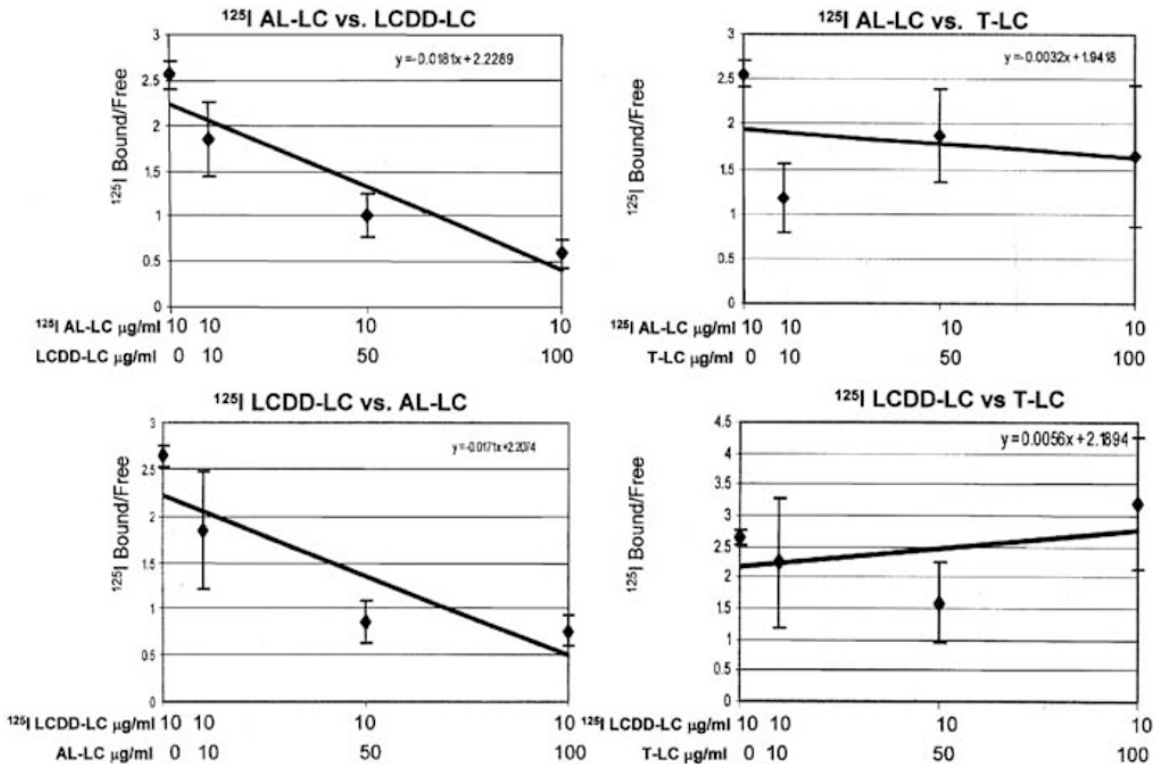

Figure $6{ }^{125} \mathrm{I}$ radiolabeled G and T-LCs incubated with HMCs for $30 \mathrm{~min}$. Competition studies with two G-LCs at the same time, one radiolabeled and the other one not radiolabeled and increasing concentrations of the non-radiolabeled LC. Competition is shown between AL and LCDD-LCs but not between AL and LCDD-LCs and T-LCs.

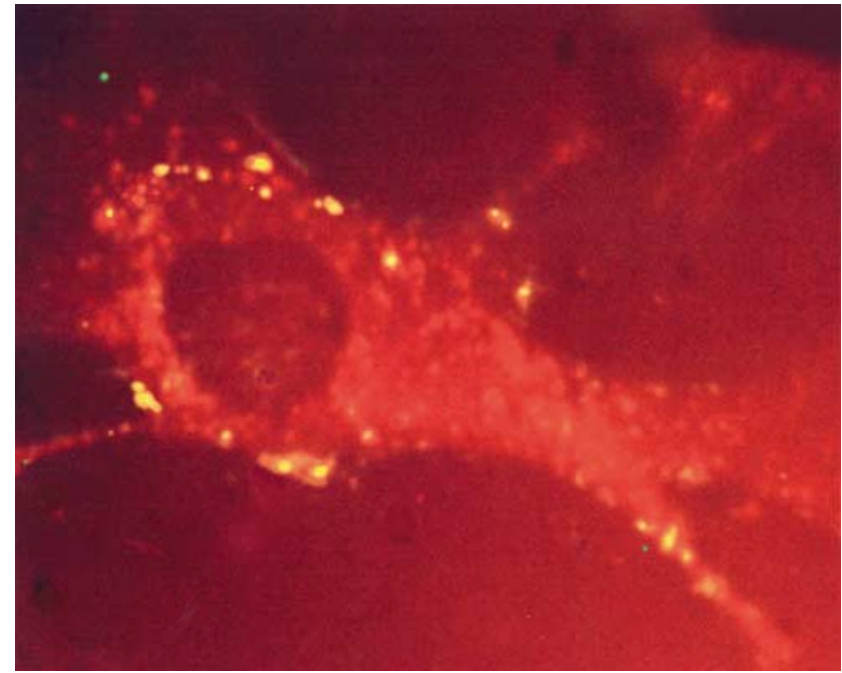

Figure 7 HMC simultaneously incubated with AL and LCDD-LCs. Immunofluorescence for AL (Texas red-tagged) and LCDD (fluorescein-tagged)-LCs. Fluorescent microscopy. Colocalization of both G-LCs represented by yellow signals is demonstrated on HMCs surface, indicating that they are both interacting with the same membrane surface receptor.

colocalization at all of clathrin with T-LCs that did not interact with MCs. Figure 9 is a schematic representation of the role of clathrin in endocytosis and intracellular protein sorting.

AL-LCs elicited avid expression of Rabs 3 and 5 at 15-30 min after incubation of HMCs with AL-LC. Later similar strong expression of Rabs 7 and 9 at 60-75 min postincubation was demonstrated (Figure 10) at the same time period that endocytosed LCs were noted to be colocalized with LAMP (Figure 11) and cathepsin D, emphasizing delivery of AL-LCs (but not LCDD-LCs) to the mature lysosomal com-

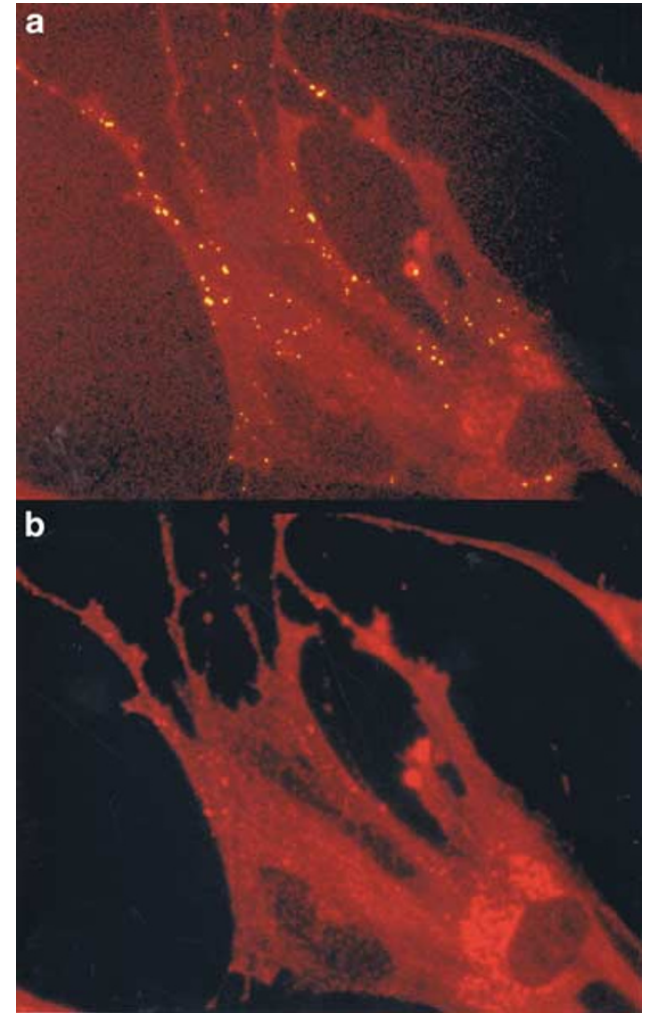

Figure 8 AX750, BX750 AL-LC incubated with HMCs. Fluorescein-tagged AL-LC (left) and Texas red-tagged clathrin (right). Note colocalization at cell surface and intracellularly, mostly close to the cell membranes (prior to endosomal processing).

partment. This did not occur with LCDD or T-LCs. LCDD-LCs only elicited Rab3 and weaker Rab 5 expression, highlighting delivery and catabolism of these LCs in the early endosomal compartment. 


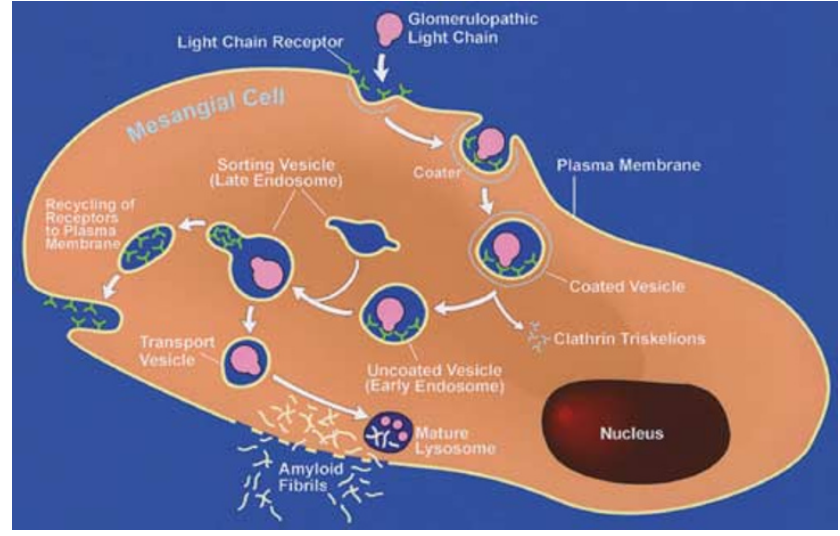

Figure 9 Schematic representation of clathrin role in LC internalization. Clathrin triskellions are detached from the LCreceptor complex prior to delivery to the early endosomes. Receptor recycling to the surface takes place during the process, as illustrated.

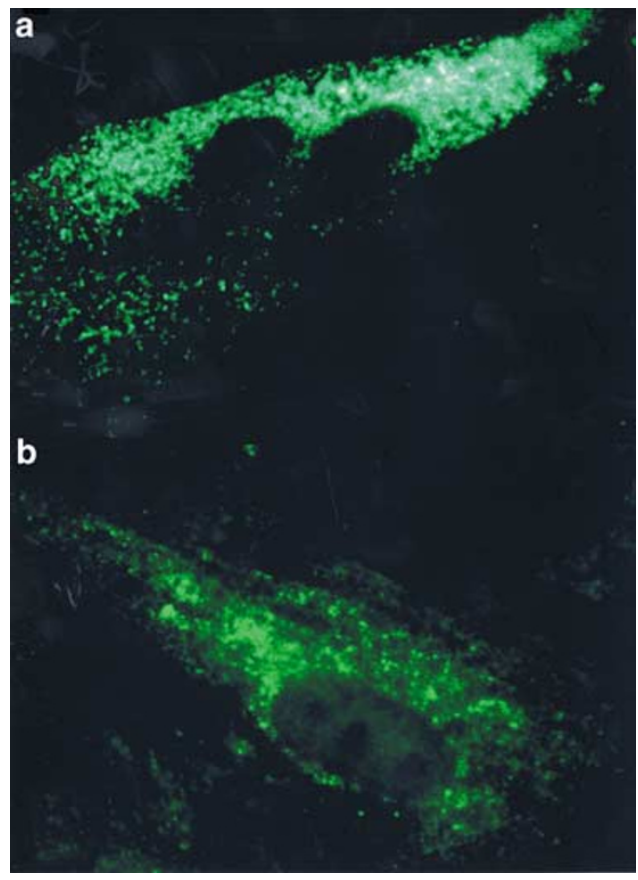

Figure 10 AX 750-BX750 fluorescein-tagged Rab 7 and 9, respectively, (a) and (b). Expression of Rabs 7 and 9 was striking in HMCs incubated with AL-LC, but not LCDD or T-LCs.

\section{Dynamic (real-time) Studies Demonstrated AL-LCs but not LCDD were Delivered to the Mature Lysosomal System}

In the case of AL-LC, the LCs were internalized avidly (Figure 12). LCDD-LCs did not show evidence of significant internalization and most of the interaction with HMCs occurred at the surface in association with the cell membranes

AL-LCs were delivered to the mature lysosomal system highlighted by LysoTracker for processing (Figure(13, top), while LCDD-LCs did not reach that compartment (Figure13, bottom), emphasizing the crucial role of the acidic milieu found in mature
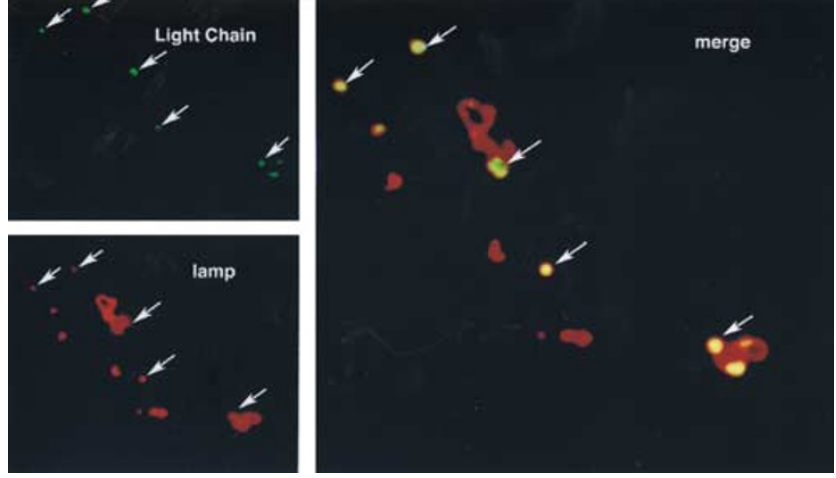

Figure 11 X750-Top, left panel-fluorescein-tagged AL-LC; X750bottom, left panel-Texas red-tagged LAMP; X750—right panel. Note colocalization of internalized AL-LC with LAMP, as shown by the yellow signal, indicating delivery of the LC to the late lysosomal compartment.

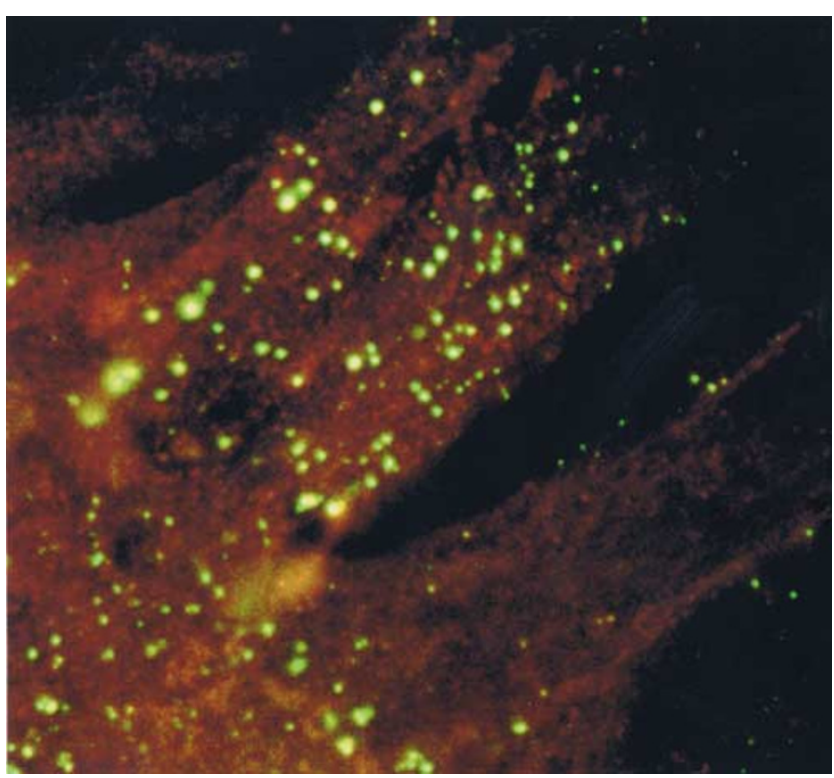

Figure 12 X750 HMCs incubated with fluorescein-tagged AL-LC. Avid internalization of AL-LC in HMCs, clearly depicted 20 min after LC incubation with HMCs.

lysosomes for amyloidogenesis. Endocytosis was most pronounced at 15-20 min after incubation of HMCs with G-LC. Real-time studies showed that the G-LCs were noted to migrate from the medium to surface areas on the HMCs. The endocytosed G-LCs were seen in the early endosomal compartment approximately $20 \mathrm{~min}$ after MCs were incubated with G-LCs. Delivery of the AL-LCs to the mature lysosomal compartment took approximately 6075 min after LC endocytosis. AL-LC processing in mature lysosomes took at least $8 \mathrm{~h}$ before amyloid was detected. Depending on the LC tested, these times noted above showed some variability, likely reflecting how avidly a given LC (due to the specific amino-acid composition of the variable region) interacted with MC receptors and generated the corresponding signals. Figure 14 shows a schematic representation of AL-LC trafficking in HMCs to reach the mature lysosomal compartment. 

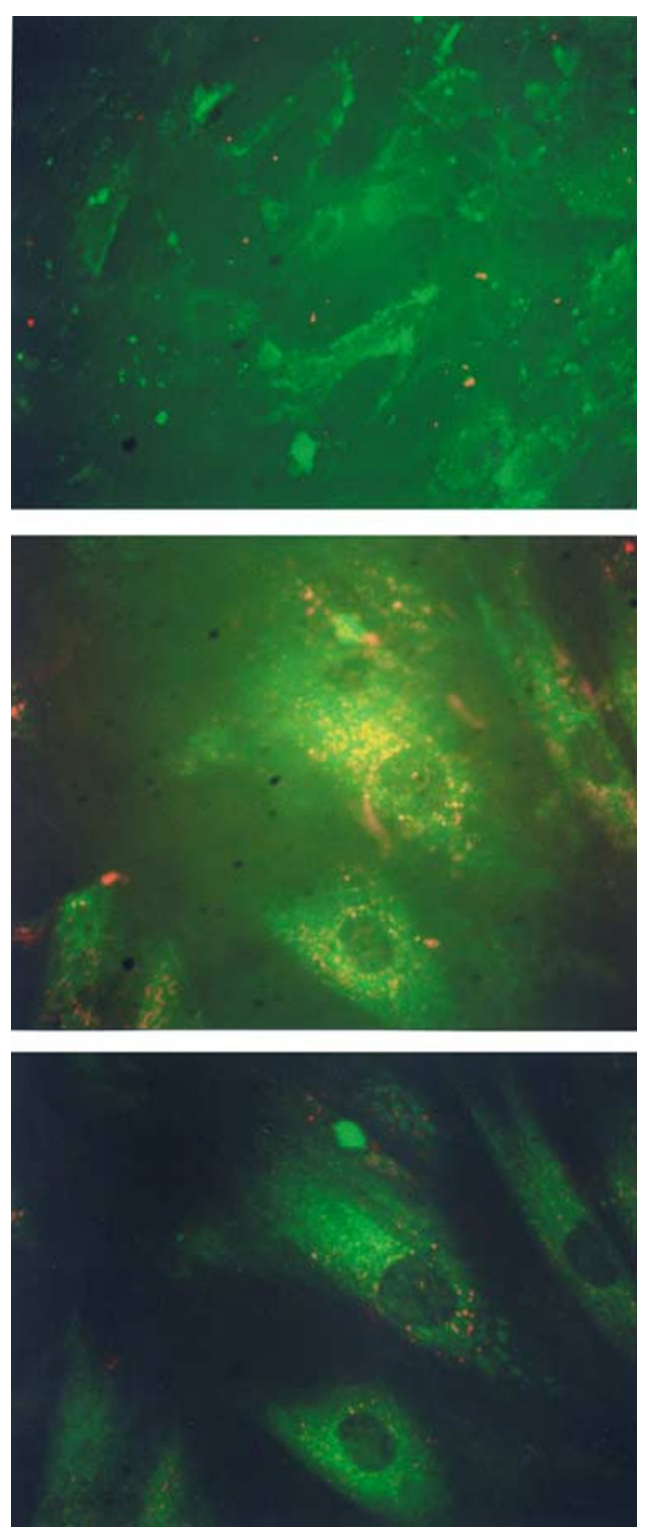

Figure 13 X500-top and middle panels and X350 bottom panel. HMCs in culture in temperature/pH-controlled chamber incubated with Texas red-labeled G-LCs (AL-top and LCDDbottom). Fluorescent microscopy. LysoTracker marking lysosomes tagged with fluorescein and G-LCs tagged with Texas red. Top$30 \mathrm{~min}$ postincubation of HMCs with AL-LC, middle-75 min postincubation with AL-LC, bottom panel-75 min postincubation with LCDD-LC. Internalized AL-LCs (top) are delivered to late (mature) lysosomal compartment where they eventually colocalize with lysosomes (middle panel-yellow indicates colocalization). In the bottom panel, note the lack of LC internalization and colocalization with mature lysosomes.

\section{G-LC-MC complex bands detected at 300-325 and 450 kDa on SDS-PAGE}

When HMCs were crosslinked to both types of GLCs (LCDD and AL-LCs), a band shift from the normal 25-30 kDa band was detected for the LCs on SDS-PAGE at approximately $300-325 \mathrm{kDa}$ (Figure 15a and b). On Coomassie blue stain, this seemingly single band (Figure 15b) resolved into two distinct bands (Figure 15c). In addition, a third band was

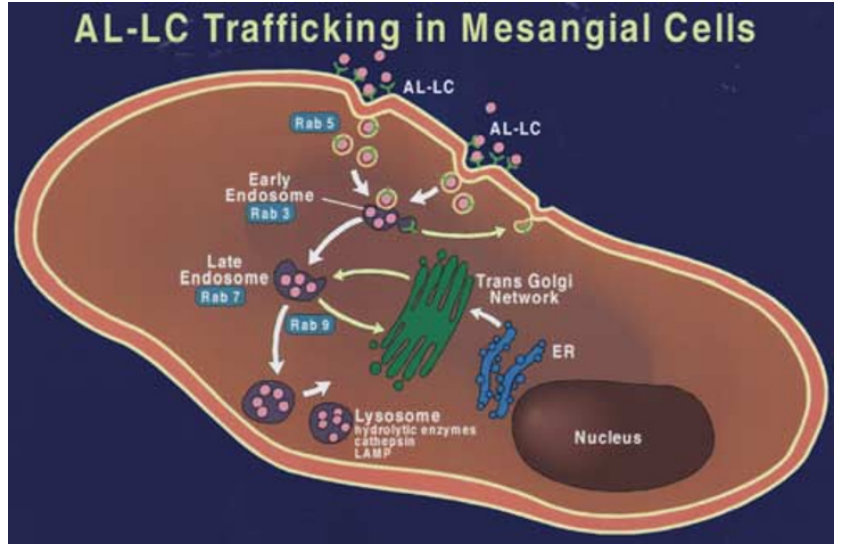

Figure 14 Schematic representation of AL-LC trafficking in HMCs. The schematic representation illustrates the intracellular trafficking of AL-LCs, as demonstrated with the dynamic (realtime) studies. As shown, there are a number of potential interventions along the way that may prevent AL-LCs from reaching the mature lysosomal compartment where amyloidogenesis normally occurs.

noticeable at approximately $460 \mathrm{kDa}$ (Figure 15). These new appearing (shifted) bands, detected to contain appropriate LC by Western blotting, corresponded to the crosslinked HMC membrane-LC complex. Mass spectroscopy of the shifted bands showed matches for filamin and talin 1, known components of caveolin-1, in the two lower molecular weight bands. There was also a weak match for kinase anchor protein in the higher molecular weight band (Figure 15d). No other matches with known receptors were detected. Determining the specific identity of the receptor is still in progress.

\section{Discussion}

Normally LCs are filtered through the glomerular capillaries and delivered to the proximal tubules where they are absorbed and catabolized. The cubilin/megalin complex receptor participates in the handling of LCs. ${ }^{11,12}$ Direct binding data suggest that cubilin first binds to megalin on the apical surface of the tubular cells membranes and the cubilin/megalin complex is then internalized simultaneously for endosomal trafficking of the endocytosed LCs. In contrast, G-LCs become entrapped in the mesangium where they exert their pathogenic effects. Defining the pathogenetic mechanisms involved in the interactions between G-LCs and MCs is crucial in order to be able to design new therapeutic strategies aimed at avoiding irreversible glomerular damage.

Normal MCs are modified smooth muscle cells and, as expected, express smooth muscle actin and muscle-specific actin. In AL-Am, the amyloidogenic LCs are avidly internalized into the MCs and delivered to the mature lysosomal compartment where amyloid formation primarily occurs. In the process, phenotypic transformation of MCs into a 
a Western blotting of Light Chain cross linking to HMC

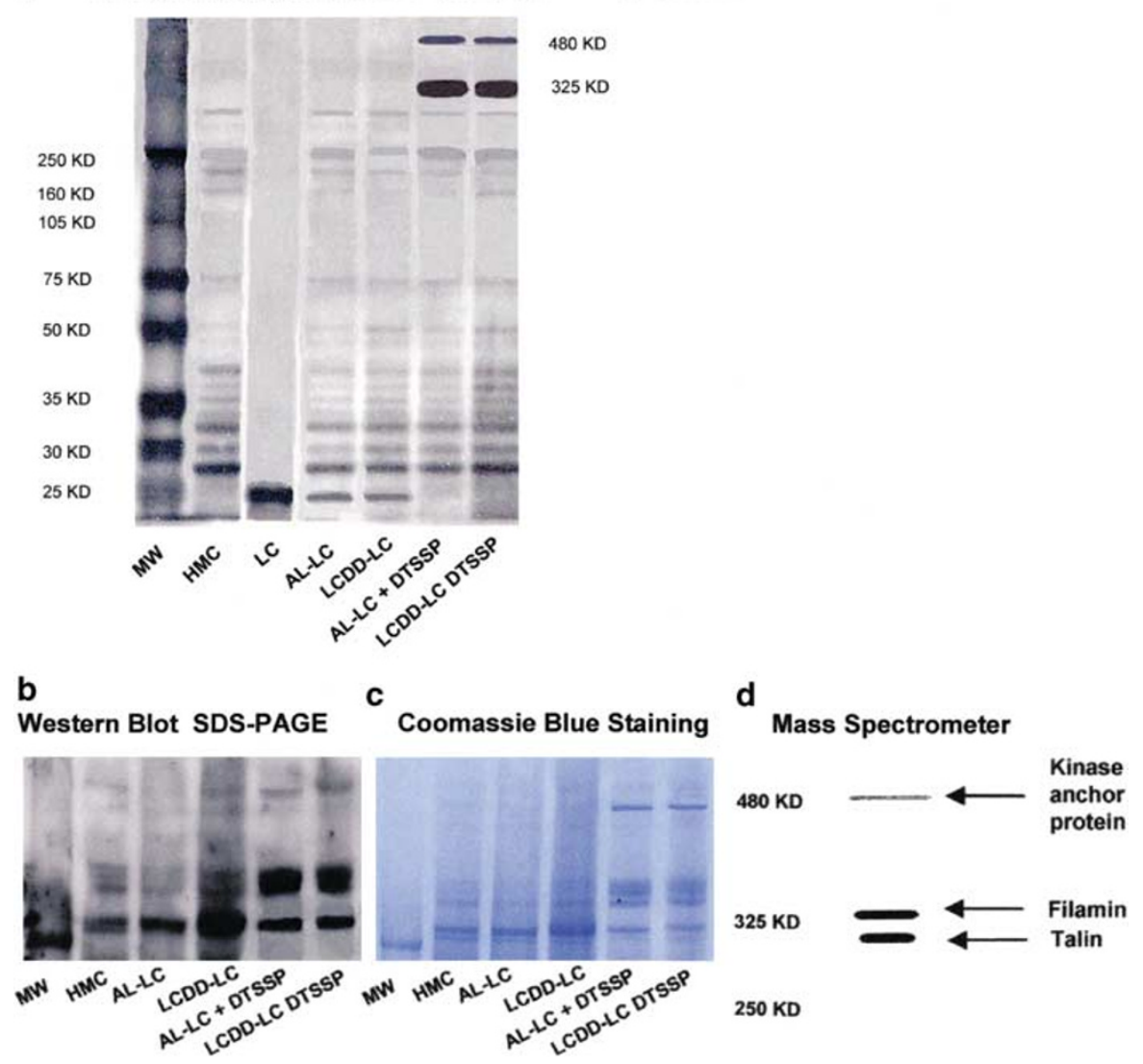

Figure 15 (a, b) Crosslinking of G-LCs to HMCs membrane. SDS-PAGE transferred to PVDF membranes. C-Coomassie blue; D-Mass spectroscopy of shifted bands. Note the shifted band containing LC (normally present at about $25 \mathrm{kD}$ ) at approximately $300-325 \mathrm{kDa}(\mathbf{a}, \mathbf{b})$ and $450 \mathrm{kDa}$ (resolved into three separate bands on Coomassie blue stain-C) representing the fragmented G-LC-MC complex. Mass spectroscopy matched filamin and talin, components of caveolin- 1 , as constituents of the shifted bands at $300-325 \mathrm{kDa}$. A weak match for kinase anchor protein was also detected in the band located at $450 \mathrm{kDa}$.

macrophage phenotype occurs (13). The transformed MCs acquire a prominent lysosomal system that makes them capable of processing internalized amyloidogenic LCs and eventually engage in the production of amyloid fibrils. The process of AL-LC internalization in HMCs and delivery to the mature lysosomal system could be elegantly shown using the controlled chamber system (Figure 13). Deposition of amyloid in the mesangium stimulates metalloproteinase activity and results in destruction of the native mesangial matrix and eventually replacement by amyloid. ${ }^{5}$ In LCDD, internalization is not an important event; the LCDD-LC-MC interactions occur at the MC surface leading to phenotypic MC alterations. In LCDD, the G-LCs-MC interactions lead to a phenotypic transformation of MCs from a smooth muscle to a myofibroblastic phenotype. The MCs acquire a prominent rough endoplasmic reticular system, which equips them to participate in active protein synthesis eventually resulting in mesangial matrix increase and formation of mesangial nodules. ${ }^{5}$ Internalization is not an important event in the interaction of LCDD-LCs with MCs, as clearly shown using the regulated chamber system.

Competition studies clearly indicate that the same receptor is involved in the G-LC-MC interactions. However, while the same receptor is involved in these interactions, the final results of the ligandreceptor interactions are diametrically opposed. The G-LC-MC interactions are key in generating the initial signaling events that eventually lead to pathologic effects. ${ }^{14}$ Colocalization studies further confirmed that G-LCs localize to the same areas on the surface of the MCs where the presumptive receptors are located.

Crosslinking studies demonstrated the presence of similar shifted bands indicative of the crosslinkage of either LCDD or AL-LCs with MCs, further supporting the notion that the same receptor is involved, regardless of the type of G-LC. Cubilin and megalin were not identified on MCs, indicating that these receptors do not mediate G-LC-MC signaling on MCs. Therefore, the receptor responsible for the 
G-LC-MC is different than that in the proximal tubular cells. The characterization of this receptor is in progress. Initial studies using mass spectroscopy have revealed the presence of filamin and talin in the displaced bands at $300-325 \mathrm{kDa}$, components of caveolin-1. ${ }^{15,16} \mathrm{~A}$ third band noted at approximately $460 \mathrm{kDa}$ demonstrated a weak match for kinase anchor proteins. It appears that the crosslinking agent used (DTSSP) may have affected the integrity of the receptor captured in the G-LC-MC complex, masquerading it. Other cross linking agents such as $\mathrm{BS}^{3}$ that do not produce cleavage may preserve the integrity of the receptor allowing proper identification. The nature of the LC receptor on MCs deserves further attention. This receptor is unique in mediating G-LC-MC interactions leading to completely opposite effects, as is the case with AL-LCs and LCDD-LCs. In the first case, the LCs are signaled to travel through the lysosomal system, eventually resulting in amyloid formation and mesangial matrix destruction with replacement of the normal matrix, while in LCDD the MCs engage in active ECM production and result in an expanded mesangium and ultimately nodular glomerulosclerosis. ${ }^{5}$

The best-characterized mechanism for endocytosis is the clathrin-coated vesicle. The crucial event in the creation of these vesicles is the recruitment of soluble clathrin from the cytoplasm into a lattice of hexagons and pentagons, forming triskelions, consisting of three copies of clathrin LC. ${ }^{17}$ In a previous publication, the mechanism of LC endocytosis, especially important in AL-LCs, was shown to be to some significant extent clathrin mediated. ${ }^{14}$ In the present study, addressing LC trafficking in MCs, it was shown that clathrin and endocytosed G-LCs colocalized. The clathrin-coated endocytosed LCs form a complex with the LC surface receptor. The clathrin triskellions are released prior to the LC delivery to the endosomes. The LC receptor is eventually recycled to the MC surface. Figure 14 shows a schematized representation of sequential events that take place.

The present study revealed cup-shaped structures on the HMC membranes with morphological features of caveolae that expressed caveolin-1. These structures increased dramatically in numbers when HMCs were incubated with G-LCs, indicating a probable function for caveolae in mediating GLCMC interactions. While caveolae are $50-80 \mathrm{~nm}$ in diameter (of a similar diameter as the surface membrane structures described above), clathrincoated vesicles are $100-150 \mathrm{~nm}$ in diameter, ${ }^{18,19}$ establishing a recognizable morphological difference among the two. Caveolae may be responsible for at least some of the nonclathrin-mediated endocytosis in cells.

The relationship between the role of caveolae with clathrin-coated vesicles in G-LC-MC binding and endocytosis remains unclear. Caveolae have been proposed to play an important role in cell surface signaling, ${ }^{19}$ in addition to endocytosis. It is probable that caveolae are essential for signaling when G-LCs contact MCs, while clathrin is more directly involved in the process of LC internalization and intracellular trafficking. The relationship between caveolae and clathrin in endocytosis and LC processing requires further investigation. Additional studies using caveolin-1 knockout mice are needed to clarify this issue. Studies performed in caveolin-1 knock out mice have shown cardiovascular defects such as aberrant arterial relaxation, contractibility, nitric oxide abnormalities, pulmonary pathology ${ }^{20}$ and physical weakness. ${ }^{21}$ It has been proposed that in the caveolin-1 knockout mice, some of the functions that caveolae normally play may be taken up by lipid rafts, ${ }^{22}$ which have also been demonstrated to be crucial in many signal transduction processes without involving caveolae. Caveolin-1, -2 and -3 represent the major constituents of caveolae. ${ }^{23}$ Caveolin-1 is expressed in two isoforms, caveolin- $1 \alpha$ and caveolin- $1 \beta$ distributed in superficial (membrane) and deep caveolae within the cells, respectively. The ratio of caveolin-1 isoforms is likely related to the degree of differentiation. ${ }^{23}$ Caveolin-1 has been demonstrated to be the most important of the three subtypes described. In vitro studies using MC cultures from caveolin-1 knockout mice should clarify some of the issues with regard to the specific functions of caveolae and their relationship to clathrin-coated vesicles.

Dynamic studies using the controlled chamber that maintains MCs physiologically viable, clearly demonstrated the fact that AL-LCs trafficked in the MCs to reach the mature lysosomal compartment, while the LCDD-LCs did not. The various cellular compartments involved in endocytosis and intracellular protein processing in mammalian cells have been elucidated. ${ }^{24-30}$ Small GTPases of the Rab family participate in and regulate intracellular protein trafficking. Rabs 3 and 5 are localized in the early lysosomal compartment (endosomes) and Rabs 7 and 9 in the late lysosomal compartment (mature lysosomes). The results noted in the trafficking and colocalization studies using Rab proteins as markers of endocytic compartments and other markers of mature lysosomes (such as cathepsin and LAMP), as well as dynamic studies in the regulated chamber, clearly indicate different patterns of LC trafficking in MCs for AL and LCDDLCs. AL-LCs are delivered to the mature lysosomal system where amyloid formation occurs, while LCDD-LCs are endosomally processed. A proposed schematic view of AL-LC trafficking in MCs is shown in Figure 14. Further studies are needed to evaluate fully the pattern of intracellular trafficking of AL and LCDD-LCs to identify the biologic importance of the differences in G-LCs processing. Additional dynamic studies performed on HMCs incubated with G-LCs can provide very useful information that will help elucidate differences in terms of how MCs process the various G-LCs. This 
will be of significance in understanding the possible pathways that can be therapeutically targeted to control or abolish increased ECM production by MCs in LCDD and amyloid formation in AL-Am.

Undoubtedly, MCs play a dominant role in progressive renal damage. ${ }^{31}$ In LC-mediated glomerular damage, the role of MCs is of paramount importance. ${ }^{2,5,13}$ The present study further advances our understanding of how G-LCs interact with HMCs to alter their behavior leading to changes in mesangial homeostasis. It also provides a conceptual platform to delineate key steps amenable to be targeted in order to control, ameliorate or abolish pathological consequences arising from the G-LCMC interactions and subsequent pathological alterations.

\section{References}

1 Verroust PJ, Birn H, Nielsen R, et al. The tandem endocytic receptors megalin and cubilin are important proteins in renal pathology. Kidney Int 2002;62:745756.

2 Herrera GA. Renal manifestations in plasma cell dyscrasias: an appraisal from the patients' bedside to the research laboratory. Ann Diagn Pathol 2000;4: 174-200.

3 Sanders PW, Herrera GA. Monoclonal immunoglobulin light chain-related diseases. Sem Nephrol 1993;13: 324-341.

4 Gallo G, Picken M, Buxbaum J, et al. The spectrum of monoclonal immunoglobulin deposition disease associated with immunocytic dyscrasias. Sem Hematol 1989;26:234-245.

5 Herrera GA, Russell WJ, Isaac J, et al. Glomerulopathic light chain-mesangial cell interactions modulate in vitro extracellular matrix remodeling and reproduce mesangiopathic findings documented in vivo. Ultrastruct Pathol 1999;23:107-126.

6 Sanders PW, Herrera GA, Galla JH. Human BenceJones protein toxicity in rat proximal tubule epithelium in vivo. Kidney Int 1987;32:851-861.

7 Harper PA, Robinson JM, Hoover RL, et al. Improved methods for culture rat glomerular cells. Kidney Int 1984;26:875-880.

8 Valenzuela R, Deodhar SD. Tissue immunofluorescence. In Rose NR, Friedman H, Fahey JL (eds). Manual of Clinical Laboratory Immunology, 3rd edn. American Society of Microbiology: Washington, DC, 1986, pp. 923-925.

9 Hsu S-M, Raine L, Fanger H. Use of avidin-biotin peroxidase complex (ABC) in immunoperoxidase techniques: a comparison between $\mathrm{ABC}$ and unlabeled antibody (PAP) procedures. J Histochem Cytochem 1981;29:577-580.

10 Herrera GA, Turbat-Herrera EA, Dell-Orto P, et al. Ultrastructural immunolabeling in renal diseases: past, present and future. Pathol Immunopathol Res 1987;6: 51-63.

11 Batuman V, Dreisbach AW, Cyran J. Light-chain binding sites on renal brush-border membranes. Am J Physiol 1990;258(Renal Fluid Electrolyte Physiol 27):F1259-F1265.
12 Batuman V, Verroust PJ, Navar GL, et al. Myeloma light chains are ligands for cubilin (gp 280). Am J Physiol 1998;275(Renal Physiol 44):F246-F254.

13 Herrera GA, Russell WJ, Cardelli J. Phenotypic transformation of mesangial cells precedes and is required for AL-amyloidogenesis. In: Bely M, Apathy A (eds). Amyloid and Amyloidosis. The Proceedings of the IXth International Symposium on Amyloidosis 2001. Budapest, Hungary, pp. 273-275.

14 Russell WJ, Cardelli J, Harris E, et al. Monoclonal light chain-mesangial cell interactions: early signaling events and subsequent pathologic effects. Lab Invest 2001;81:689-703.

15 Stahlhut M, Deurs B. Identification of filamin as a novel ligand for caveolin-1: evidence for the organization of caveolin-1-associated membrane domains by the actin cytoskeleton. Mol Biol Cell 2000;11:325-337.

16 Cantarelli VV, Takahashi A, Yanagihara I, et al. Talin, a host cell protein, interacts directly with the translocated intimin receptor, Tir, or enteropathogenic Escherichia coli and is essential for pedestal formation. Cell Microbiol 2001;3:745-751.

17 Robinson M, Watts C, Zerial M. Membrane dynamics in endocytosis. Cell 1996;84:13-21.

18 Anderson RG. The caveolae membrane system. Annu Rev Biochem 1998;67:199-225.

19 Parton RG. Caveolae and caveolins. Curr Opin Cell Biol 1999;8:542-548.

20 Drab M, Verkade P, Elger M, et al. Loss of caveolae, vascular dysfunction and pulmonary defects in caveolin-1 gene disrupted mice. Science 2001;293: 2449-2452.

21 Parton RC. Life without caveolae. Science 2001;293: 2404-2405.

22 Galbiati F, Razani B, Lisanti MP. Emerging themes in lipid rafts and caveolae. Cell 2001;106:403-411.

23 Fujimoto T, Kogo $\mathrm{H}$, Nomura $\mathrm{R}$, et al. Isoforms of caveolin-1 and caveolar structure. J Cell Science 2000;113:3509-3517.

24 Bottger G, Negelkerken B, van der Sluijs P. Rab 4 and Rab 7 define distinct non-overlapping endosomal compartments. J Biol Chem 1996;227:29191-29197.

25 van der Sluijs P, Hull M, Webster P, et al. The small GTP-binding protein rab4 controls an early sorting event on the endocytic pathway. Cell 1992;70:729-740.

26 Merese S, Gorbel JP, Chavrier P. The Rab 7 GTPase resides on a vesicular compartment connected to lysosomes. J Cell Science 1995;108:3349-3358.

27 Feng Y, Press B, Wandinger-Ness A. Rab 7, an important regulator of late endocytic Membrane traffic. J Cell Biol 1995;131:1435-1452.

28 Press B, Feng Y, Hoflack B, et al. Mutant Rab 7 causes the accumulation of cathepsin $\mathrm{D}$ and cation independent mannose-6 phosphate receptor in an early endocytic compartment. J Cell Biol 1998;140: 1075-1089.

29 Vitelli R, Santillo M, Lattero D, et al. Role of small GTPase Rab 7 in the late endocytic pathway. J Biol Chem 1997;272:4391-4397.

30 Schmid SL, Sorkin AD. Days and knights discussing membrane dynamics in endocytosis: meeting report from the Euresco/EMBL membrane dynamics in endocytosis. Traffic 2002;3:77-85.

31 Striker LJ, Doi T, Elliot S, et al. The contributions of glomerular mesangial cells to progressive glomerulosclerosis. Semin Nephrol 1989;9:318-328. 